\title{
A diagrammatic approach to computer instruction for the naive user
}

\author{
MARC M. SEBRECHTS and JOSEPH G. DECK \\ Wesleyan University, Middletown, Connecticut 06457 \\ and \\ JOHN B. BLACK \\ Yale University, New Haven, Connecticut 06520
}

\begin{abstract}
There are inherent difficulties with text as an instructional format. This research presents the development of a diagrammatic representation that overcomes some of those difficulties. A simple "language" is presented that includes both procedural and model information. This approach is applied to the instruction of word processing/text-editing skills. An experimental methodology is presented and preliminary results are reported that suggest that the diagram. matic approach results in improvements in both the amount of word processing accomplished and the speed with which modifications are made.
\end{abstract}

With rapid changes in computer technology, it has become extremely important to make systems easy to use by a rather large group of people. There are numerous ways to do this, including major changes in system design and changes in the types of instructional and documentation materials available to users.

Although the problem is evident for users with varying amounts of experience, the difficulties are particularly striking for the naive user who has had little if any prior computer experience. For the uninitiated, the information available on a new computer can resemble a foreign language, with the corresponding misinterpretations. The research described here is directed toward decreasing the alien character of instructions for this group.

Our strategy in doing this has been to develop a set of cognitive principles that can serve as the basis for instructional design (Black \& Sebrechts, 1981). We are developing these principles by an experimental evaluation of word processing/text-editing skills. Specifically, we are interested in designing and assessing a diagrammatic "language" for computer use. Although in these studies we are focusing on word processing instruction, we believe that the same principles should be useful for both instruction and documentation of a wide class of complex skills.

In this paper, we will provide a general description of our assessment of a diagrammatic language for instruction. First, the conceptual motivation is described for the use of a diagrammatic language. Then, the structure of the language is presented, with the underlying cognitive rationale. Finally, we present our strategy for

The research described in this paper was funded in part by a contract with the IBM Corporation. evaluating this language experimentally and provide some preliminary results of the investigation.

\section{INSTRUCTIONAL OBJECTIVES}

When faced with a novel task, users require instruction. Frequently, people are reluctant to use instructions as they are currently presented (Lewis \& Mack, 1982), but a computer environment is far too complex to hope that users will understand the system by learning through induction. While we do not think that it is reasonable to assume that instruction can be eliminated, we do think that there are major problems inherent in most standard textual instruction manuals.

On the one hand, an instruction manual is designed to tell the user how to accomplish each task. The user wants to know the specific procedures that must be followed for any task and is likely to ask questions such as "What keys do I need to type?" or "What is the name of the next command?" In order to learn the answers to these types of questions, novices frequently request that we minimize the descriptive information. At this point, the user wants a cookbook without adornment. Extended text describing relations within the system can frequently lead to frustration. Individuals have limited resources to allocate to a task (Norman \& Bobrow, 1975), and the presentation of enormous amounts of information can lead to an overload of those capacities. For many people, procedures are often best described by simply laying out the appropriate steps to follow, without "superfluous" commentary.

However, in the case of complex systems like a computer, users quickly run into difficulty if they are given only specific procedures. Naive users do not understand the relations between components of the system, and 
they therefore often construct mental models that are completely inappropriate for the context in which they are working (Halasz \& Moran, 1982). One rather striking example is presented by Bott (Note 1), who indicates that some subjects understand the PRINT command to refer to the context of a printing press. More generally, people make errors by misunderstanding the context in which they are operating. These are labeled "mode" errors (Norman, 1981). For example, if subjects are using the UCSD PASCAL operating system (Softech Microsystems, 1980) and they want to change the name of a file, they may type the reasonable mnemonic "c" for "CHANGE." This would in fact be exactly the right action to take if the user were in the "Filer mode." However, if the user is in the "Command mode" instead, typing a "c" is the appropriate mnemonic for "COMPILE."

When faced with these confusions, subjects need additional information about the overall design of the computer system, including a description of relations among the various commands and menus. Of course, this type of relational information requires substantial additions of text, in direct conflict with the previous goal of eliminating text in order to highlight the procedures.

\section{THE MOTIVATION FOR A DIAGRAMMATIC LANGUAGE}

Given these conflicting goals, it is difficult to imagine how we could achieve an optimal text. We are simultaneously trying to minimize the amount of text in order to make the procedures stand out clearly and to maximize the descriptive context in order to help the user develop a mental model of system structure.

In order to solve this problem, we have devised an alternative diagrammatic "language" for instruction. The key element in this alternative approach is the use of a spatial layout as an additional dimension for capturing system information. Spatial relations can be used to convey information about relational properties, as well as to provide groupings that highlight specific procedures.

In formulating a diagrammatic language that will provide this added informational dimension, we have drawn on work in memory, imagery, and perception. Much of the research on spatial cognition has focused on the ways in which internalized spatial representations capture descriptions of physical space. The research described here is designed to illustrate that the "isomorphic" mapping of external space onto an internal cognitive map is only one kind of mapping in which a spatial description is useful; diagrammatic descriptions need not depend directly on an inherently spatial domain but can in fact be used for nonspatial information as well.

The intuition behind this approach is captured in the research on different types of cognitive maps.
Trying to explain a complicated set of directions verbally can be extremely difficult, and the task can be substantially facilitated by using visual aids. In order to better comprehend how this same facilitation might operate in the domain of instructional materials, we will briefly examine the distinction between two types of maps, the route map and the survey map.

A route map provides a specification of the route that is to be followed. In essence, this type of map indicates how to get from one location to another by following a specific set of directions. The route map only lays out the route, and if you become lost, there is relatively little hope of recovery. In contrast, a survey map describes "the lay of the land." It provides a general description of an area, with many different routes, including the specific one that is to be followed. Here, the emphasis is on the context between two locations. Although different routes are available, none of the specific routes is necessarily highlighted.

We have taken this map metaphor as a basis for capturing the types of information that are relevant to a complex procedural task. On one hand, route maps can be thought of as providing a description of the specific script-like procedures that are to be followed. Survey maps, in contrast, make it possible to determine relationships among components and can therefore serve as the basis for an overall model or "frame."

Given these two types of information, there are several ways to use them for instruction. Research on cognitive maps suggests one way people learn (Hart \& Moore, 1973). Children begin with route maps and later acquire survey maps. Adults show a similar trend in their learning patterns of new areas. When they begin a job in a new city, for example, they may know how to get from home to work following a particular route. With time, however, they learn many possible alternate routes and discover more general geographical relationships between their home and place of work. We can think of an analogous situation in the domain of nonspatial skills. In the course of learning about a domain, we take what are essentially rather rigid scripts (Schank \& Abelson, 1977) and embellish them within the context of more flexible actions.

The transition from the rigid procedure to a more flexible set of alternatives, however, can be quite difficult. Thorndyke and Hayes-Roth (1982), for example, examined the knowledge that secretaries had of the Rand building, in which they worked. Although they could all describe how to get from one place to another, only after they had been at Rand for about 10 years were they able to indicate the geographical locations of two specified locations, such as the photocopy room and the lunchroom. Presumably, a great deal of integration is required to put the route maps into a survey map context.

To function effectively, people need general memorial models together with their detailed procedures or 
scripts. This is a notion perhaps best caught by Minsky's (1975) notion of frames or Schank's (1981) notion of memory organization packets (MOPS). In both of these cases, specific details are integrated into a more general structure that can call upon the individual procedures.

We do know that in the context of word processing skills, people make many inferences and structure relationships, only some of which are correct. In the terms of Carroll and Mack (Note 2), people are active learners. Given that people are actively building mental models, how can we direct that active learning? Our strategy is to integrate both procedural and model information into what we will call a contextualized route map. In other words, we need both procedural and model information. This can be achieved by utilizing the following modified forms of route and survey maps: (1) A specific description is given of the steps in a procedure, in a way analogous to a route map. (2) The procedure is put within a local context, providing some error recovery options, as with a survey map. (3) The

VIEWING A DOCUMENT ON THE DISPLAY

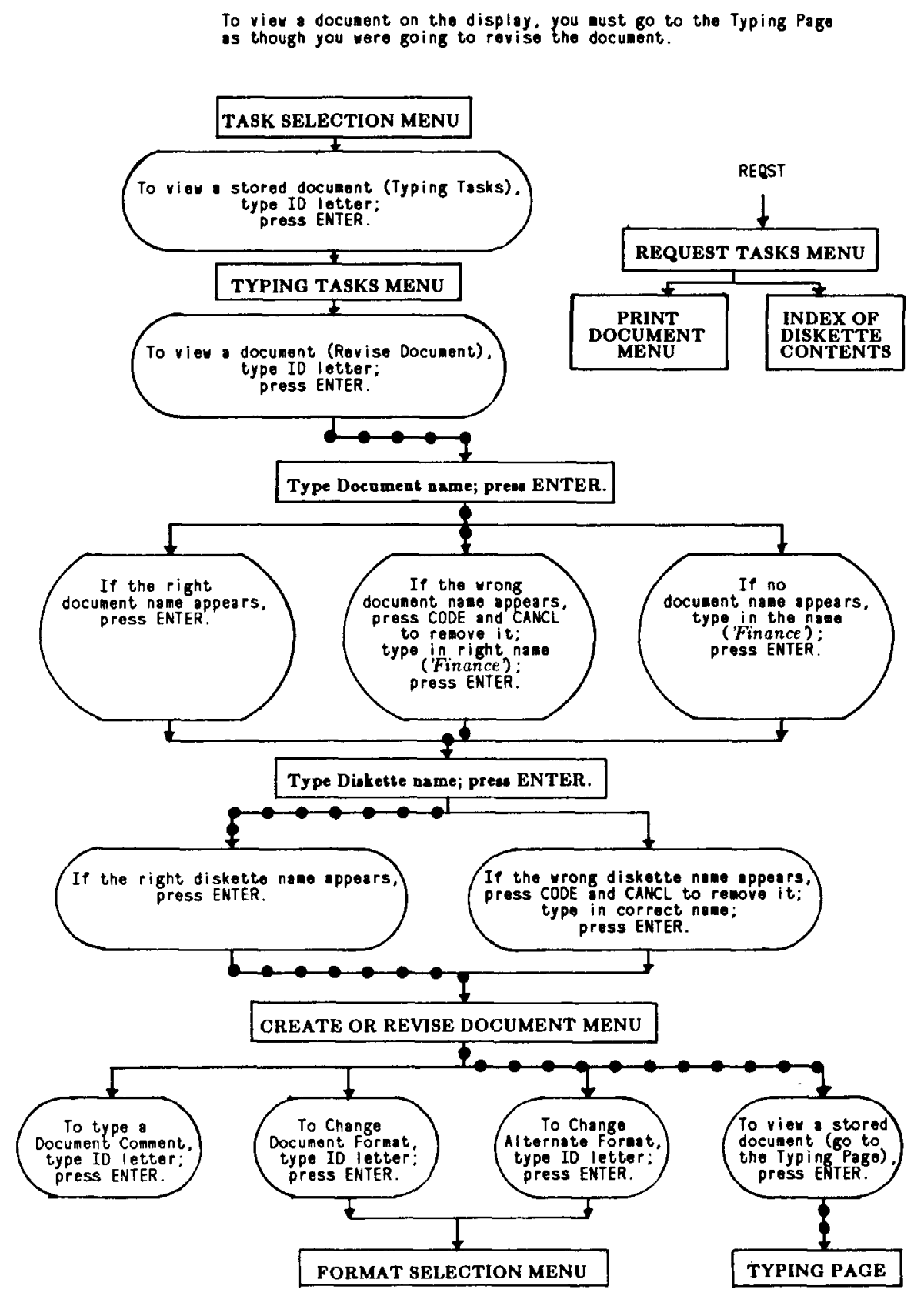

Figure 1. Modified DIAGRAMMATIC instructions indicating how to view a document (file) on the IBM Displaywriter. (From International Business Machines, 1982; reprinted by permission.) 
Each time you create a file (type a new test) you will need a new workspace. The new file is empty until you type some text into it.

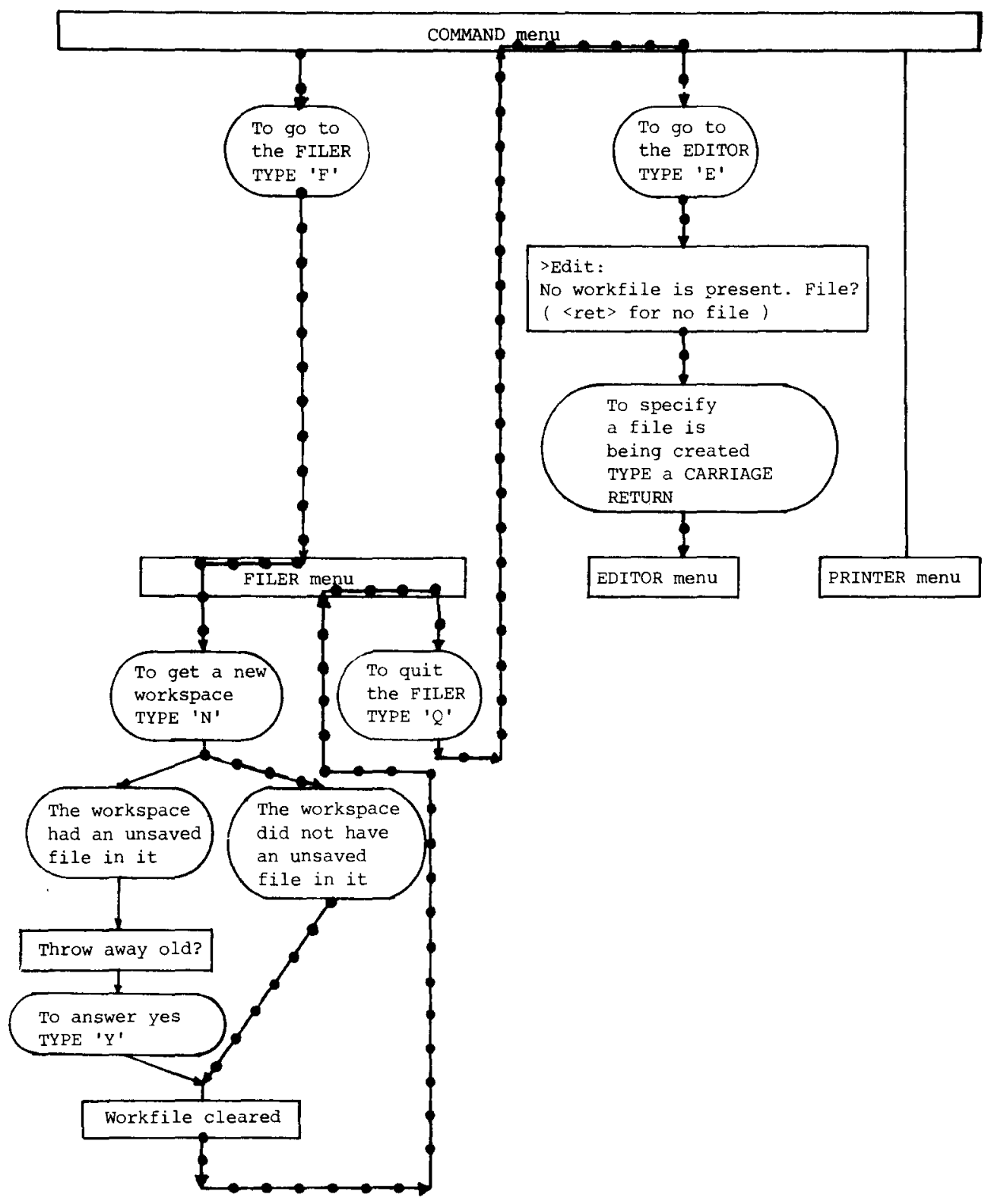

Figure 2. Modified DIAGRAMMATIC instructions indicating how to create a file using UCSD PASCAL. The instructions are matched with those in Figure 4.

procedure is embedded in a general framework by providing a schematic survey map (SKELETON) of the major components of the system.

The basic strategy, then, is to utilize the spatial domain in a way analogous to the use of maps, in order to provide both mental models (a survey map or frame) and procedural descriptions (a route map or detailed script). Examples of the results of this diagrammatic approach are shown in Figures 1 and 2, for viewing a document (file) on the Displaywriter and creating a file in UCSD PASCAL, respectively. We will describe some additional constraints on these diagrams before discussing them in detail.

\section{MODULARIZATION}

Implementing a description of the system with a series of "maps" would be extremely unwieldy, like trying to have all the street maps of the United States on one giant page. In the case of road maps, this problem is 
solved by delimiting certain spatial boundaries for each separate map. In the case of instructions, an attempt is made to break the information into conceptual chunks. Other research has shown the importance of organization for words (Bower, Clark, Lesgold, \& Winzenz, 1969) or common activities (Bower, Black, \& Turner, 1979). In the case of spatial memory, visual organization is important. Thorndyke and Stasz (1980) collected verbal protocols from subjects while they were learning a map. One important strategy employed by the subjects consisted of mentally partitioning the map into subunits; this strategy was used more by the good learners than by the poor learners.

In the cognitive map studies, partitioning was done by region. In the case of diagrammatic instructions, partitioning needs to be done on the basis of meaningful subtasks. In order to do this, we divided the instructions into a set of modular tasks, such as SAVE a file or PRINT a file. Insofar as possible, all materials relevant to a task, including commands and menus, were presented on a single page.

\section{CONCEPTUAL ZOOMING}

A second basic issue in this diagrammatic language concerns the information that is to be presented for each modular task. As noted above, we want to capture both the specific detail of procedures and the basic relationships among procedural units. One advantage of the spatial domain in this respect is that it is possible to present a clear hierarchical structure between the overall system structure (diagrammatic frame) and procedural information (diagrammatic detail).

The description of this type of visual scene analysis has been advanced by Kosslyn and his colleagues (Kosslyn, Pinker, Smith, \& Schwartz, 1979; Kosslyn \& Schwartz, 1977) as part of a theory of imagery. In the imagery case, for example, we can construct a mental image of an elephant. This image will give us an overall description of the elephant and general information about presence or absence of tusks, rough size of the ears, and so forth. If we want to see the skin texture on the trunk, however, we need to expand that portion of the image. We step down in the hierarchy by "zooming in" on the mental image.

In the case of learning word processing, a type of analogous conceptual zooming is provided. In Figure 3,
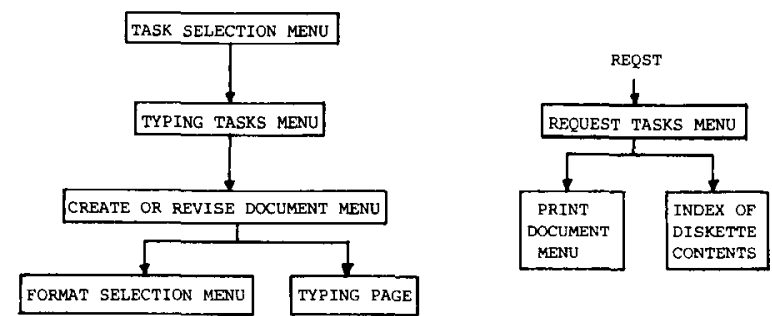

Figure 3. This SKELETON describes the relations between menus and was used in all Displaywriter diagrams. (From International Business Machines, 1982; reprinted by permission.) for example, only the skeletal structure for the IBM Displaywriter is provided. Figure 1 shows a detailed development of one section of that chart, not present in the skeletal model. We begin with a general description of the system; in order to execute a specified task, we then zoom in on specific procedures as needed. However, one needs to avoid losing the forest for the trees, or the elephant for the trunk. In the imagery case, it is probably sufficient to retain a link from the image of the trunk to "elephant," and the superordinate image can be reconstructed at will. In the computer case, the issue is somewhat different, since the user cannot retrieve the system and frequently is unaware of where a procedure is located within the overall system.

Therefore, we need to preserve the overall structural information of the system, while at the same time providing sufficient detail at the level of the specific procedures currently being conducted. To provide this type of information, the diagrammatic descriptions always maintain a general descriptive picture of the key features of the system. This central structure provides a SKELETON of the overall system, which informs the user of their general location. (The skeleton for the IBM Displaywriter is shown in Figure 3.) The idea behind the skeleton is that we need to preserve for the users the overall structure of the system without providing a lot of irrelevant detail, thus helping them to focus their attention on the most important procedural aspects of their task.

However, a very detailed description of the particular procedures that are currently relevant to the user is needed. We thus have detailed ACTIVE PROCEDURES. It is here that the user will need maximum information. In addition to providing the specific procedure that the user is to follow, however, we maintain a context in keeping with the notion that our route maps should be embedded in the context of the survey maps. Figure 1 illustrates an attempt to achieve this objective in a specific case for the Displaywriter. The task here is to view a document on the screen that has been previously edited. The diagram includes the principal menus from the skeleton in Figure 3. The Request Task Menu section on the right remains as it was in the skeleton, since it is not being used; it serves only as a reference point. The other parts of the skeleton are also present, but there is an expansion of the procedural description starting at the Task Selection Menu. The diagram includes alternative procedures in each active region used. At the bottom, the Format Selection Menu remains unelaborated, since it is not used here; it too serves as part of the skeletal reference framework.

\section{SEMANTIC AND SYNTACTIC COMPONENTS OF DIAGRAMS}

Thus far, the general properties for a diagrammatic "language" for instruction have been described. There are two basic components of this language that are used to capture both models and procedures. First, specific 
descriptions (route maps) are provided for procedures, and these procedures are presented in the context of an overall model (survey map). Second, greater detail is provided for those portions of the procedures that are active at a given time, and only a skeleton remains for the inactive descriptions.

In order to embody these general principles, we need to clarify the symbols used to differentiate the components. In the diagrams, boxes indicate system information; they represent menus or prompts presented on the screen to the user. Ovals indicate user information; they contain the possible goals and the actions required to accomplish those goals. In general, the description of a movement from one portion of the system to another requires an oval between boxes. The control flow between the various menus and user actions is indicated by directed lines. The specific procedure that a user is supposed to follow in completing the tutorial is indicated by the fact that the control flow lines are highlighted in red (indicated in the figures by dotted lines). The representation has been kept deliberately simple in order to minimize the learning that a user would need in order to master the use of the diagrams.

\section{EXPERIMENTAL EVALUATION}

\section{Materials}

The diagrammatic representation that we have described does not fully constrain how instructional materials should be made. This lack of constraint is deliberate; the diagrammatic representation needs to match the characteristics of the particular computer for which it is being used. The psychological import of matching a representation to the domain it is intended to represent has been demonstrated by Durding, Becker, and Gould (1977). They found that people could easily fit words into a skeletal structure that explicitly maintained semantic relations among words, although they had great difficulty fitting those same words to a skeletal structure that subjects perceived as "inappropriate." For example, if a college curriculum had predefined hierarchical semantic relations, subjects could easily fit courses into a hierarchy, but not into a network.

In order to provide some generality, we have developed diagrams for three systems: a portion of the UCSD PASCAL operating system on the TERAK (Softech Microsystems, 1980), the WordStar word processing system (MicroPro International Corporation, 1980), and the IBM Displaywriter (International Business Machines, 1981). Each system has its own peculiar characteristics, which we have tried to capture within the constraints of our diagrammatic language. UCSD PASCAL utilizes a rather straightforward hierarchical menu structure; a command can be reached only from a superordinate level, making it necessary to return to that level to follow another branch. The Displaywriter structure is somewhat more complicated. In general, there is still a hierarchical structure, but there are several different ways to move between menus, making the structure a heterarchy. This relation can be seen in Figure 1; the REQUEST MENU is set off to the side, since it can be reached directly by the request key. The WordStar system can also be thought of as a hierarchy, but that structure does not capture the basic conceptual organization. Most of the basic editing functions (e.g., FIND, INSERT, DELETE) are performed from a MAIN menu. However, special functions, such as formatting or printing, are relegated to separate menus. Usually, when an action is performed in one of these related menus, the subject is returned directly to the MAIN menu. These relationships seem to be best captured by a satellite structure, with the MAIN menu in the center and other menus branching to the side in a star-like pattern.

As a test of the diagrammatic language, two sets of parallel manuals were developed for each of the three systems (Displaywriter, UCSD PASCAL, WordStar). One manual was a text version (based on International Business Machines, 1981, for the Displaywriter and on Etlin, 1981, for WordStar); a second manual was developed for each system using the diagrammatic format, matched for content as closely as possible to the text version. Examples of both the text and the diagrammatic versions for the UCSD PASCAL manual are shown in Figures 2 and 4 . Both versions specify the creation of a letter. They have been matched in terms of goals and actions. The text version (Figure 4) refers to the menus whenever a change is made from one menu to another; these same changes are presented spatially in the diagrammatic version (Figure 2).

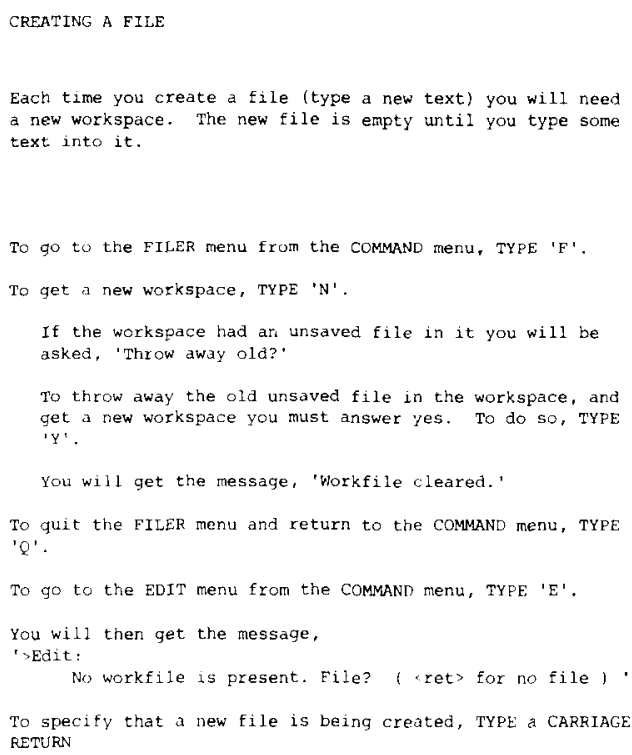

Figure 4. The TEXT instructions indicating how to create a letter using UCSD, PASCAL. The instructions are matched with those in Figure 2. 


\section{Experimental Design}

For each of the three computer systems, we trained and tested subjects on basic word processing skills. Each subject participated in five sessions lasting approximately $2 \mathrm{~h}$ on each of 5 consecutive days. On the 1 st day, subjects were given a battery of abilities tests, a similarity scaling, a typing test, and a computer knowledge questionnaire. On the 2nd and 3rd days, subjects learned basic word processing by using either the text or the diagram manual. An experimenter was present and performance was videotaped, but the experimenter intervened only if the subject was unable to continue using the manual without assistance. During the training, subjects learned how to type in a file, save a file, retrieve a file, edit a file, and print a file. All subjects were required to go through all of the tasks.

The training was followed by two testing sessions in which subjects were required to perform a series of typical editing tasks. The tasks in the two sessions were equated for length of files, the relative location of errors in the files, and the types of errors to be corrected in the files. In the first session, however, subjects were not permitted to use the manual and had to rely on their memory; in the second session, subjects were permitted to refer to the instructional manuals. This provided a way to assess the subjects' reliance on the instructional materials.

In each session, subjects were given four tasks: (1) type and print a one-page memo, (2) edit, save, and print a stored file, (3) rearrange items in a stored file, save the file, and print it, (4) edit a stored file using commands specified by the experimenter and save the revised file. The first three of these tasks were used to assess how well subjects would do on usual word processing tasks. The second task examined a user's ability to make corrections using simple cursor movement and text-modification commands. The third task required more "advanced" skills, including the modification of invisible characters. The fourth task tested directly the subjects' knowledge of individual commands, which they may know but fail to utilize unless they are required to do so. At the end of testing, subjects were again required to complete a questionnaire and provide similarity ratings for various commands.

\section{PRELIMINARY RESULTS}

Our experimental design is a mixture of constrained laboratory research and "ecologically valid" assessment. People are given a task that is similar in many respects to typical word processing instructions, although we have introduced a number of additional constraints on what they can use in trying to accomplish their tasks. We have also utilized measures of individual difference and probes of users' conceptual models.

Here, the focus is on the effects of the training conditions (textual or diagrammatic) on overall word processing skills. To evaluate users' ability, we devised a series of measures of performance in the final tests.
These included measures of both accuracy (how much subjects accomplished in each of the tasks) and speed (how long it took to complete the modifications).

The preliminary results are based on UCSD PASCAL. Across the four tasks presented, subjects performed better with the diagrammatic manual. Differences were quite consistent for a number of different measures and different tasks. The pattern was also similar for the 2 days of testing, although subjects generally did better on the 2nd day. In general, subjects made relatively little use of the manual during testing. The pattern of results can be summarized fairly well by collapsing across the four tasks for both days, which we have done for the following results. The editing tasks included a number of steps for each modification, whereas the movement between various menus usually required only a single step. We have therefore separated the editing tasks from the system tasks, which include the filing and printing tasks.

Subjects accomplish about $12 \%$ more of the system functions and $23 \%$ more of the editing functions when using the diagram manual than when using the text manual. This can, in part, be attributed to the fact that the subjects in the diagrammatic condition simply attempted more tasks. However, there is still an advantage for the diagram group when we take this difference into account. The diagram group accomplished about $6 \%$ more of the system tasks they attempted and about $21 \%$ more of the editing tasks they attempted than the text group did.

The diagrammatic group was also generally faster in making modifications. Modifications are actually performed rather quickly; on average, it takes about $30 \mathrm{sec}$ to accomplish a system command, and about $135 \mathrm{sec}$ $(2.25 \mathrm{~min})$ to accomplish a modification in the editor. Nevertheless, taking the mean time for accomplishing commands in each of the tasks, the diagrammatic group performed system commands about $43 \%$ faster and editor commands about $28 \%$ faster than the text group did.

These results are preliminary and should be treated accordingly. However, the overall pattern of results does suggest that the diagrams did improve tutorial learning. In addition, we are encouraged by another informal assessment of the manuals. Following the testing sessions, subjects were given both the manual they had used and the alternative version of the manual and were asked which one they preferred. In all cases, subjects preferred the diagrammatic over the textual version of the manual. Given the difficulty frequently reported in getting subjects to use the manuals, this may make an important difference at a motivational level.

\section{SUMMARY}

We have described our initial studies in designing a diagrammatic language for instruction of word processing/text-editing skills. A general theoretical framework for learning complex skills has been indi- 
cated, in which it is important to present procedural information within the context of a model of the system. The described experimental procedure is designed to evaluate our initial implementation of this diagrammatic language in three systems. Initial results on one of these systems suggest that our approach results in improved speed and accuracy on a number of editing tasks when compared with a textual approach.

\section{REFERENCE NOTES}

1. Bott, R. A. A study of computer learning: Theory and methodologies (Tech. Rep. 82). San Diego: University of California, Center for Information Processing, 1979.

2. Carroll, J. M., \& Mack, R. L. Learning to use a word processor: By doing, by thinking, and by knowing (Tech. Rep. RC 9481). Yorktown Heights, N.Y: IBM Watson Research Center, 1982.

\section{REFERENCES}

Black, J. B., \& Sebrechts, M. M. Facilitating human-computer communication. Applied Psycholinguistics, 1981, 2, 149-177.

Bower, G. H., Black, J. B., \& Turner, T. J. Scripts in memory for text. Cognitive Psychology, 1979, 11, 177-220.

Bower, G. H., Clark, M. C., Lesgold, A. M., \& Winzenz, D. Hierarchical retrieval schemes in recall of categorical word lists. Journal of Verbal Learning and Verbal Behavior, 1969, 8, 323-343.

Durding, B. M., Becker, C. A., \& Gould, J. D. Data organization. Human Factors, 1977, 19, 1-14.

Ethin, W. A. WordStar made easy. Highstown, N.J: OsborneMcGraw, 1981.

Halasz, F., \& Moran, T. Analogy considered harmful. In Proceedings of Human Factors in Computer Systems. Washington, D.C: Association for Computing Machinery, 1982.
Hart, R. A., \& Moore, G. I. The development of spatial cognition. A review. In R. M. Downs \& D. Stea (Eds.), Image and environment. Chicago: Aldine, 1973.

International Business Machines. IBM Displaywriter system operator training book $1-$ Basic topics. Austin, Tex: Author, 1981.

International. Business Machines. Improving the ease of learning to use a system. Austin, Tex: Author, 1982.

Kosslyn, S. M., Pinker, S., Smith, G. E., \& Schwartz, S. P. On the demystification of mental imagery. Behavioral and Brain Science, 1979, 2, 535-587.

Kosslyn, S. M., \& Schwartz, S. P. A simulation of visual imagery. Cognitive Science, 1977, 1, 265-298.

LEwIS, C., \& MACK, R. Learning to use a text-processing system: Evidence from "thinking aloud" protocols. In Proceedings of Human Factors in Computer Systems. Washington, D.C: Association for Computing Machinery, 1982.

MicroPro International. Corporation. Wordstar user's guide. San Rafael, Calif: Author, 1980.

Minsky, M. A framework for representing knowledge. In P. H. Winston (Ed.), The psychology of computer vision. New York: McGraw-Hill, 1975.

Norman, D. A. Categorization of action slips. Psychological Review, 1981, 88, 1-15.

Norman, D. A., \& Boвrow, D. E. On data-limited and resourcelimited processes. Cognitive Psychology, 1975, 7, 44-64.

Schank, R. C. Language and memory. In D. A. Norman (Ed.), Perspectives on cognitive science. Norwood, N.J: Ablex, 1981.

Schank, R. C., \& Abelson, R. P. Scripts, plans, goals and understanding. Hillsdale, N.J: Erlbaum, 1977.

Softech Microsystems. UCSD PASCAL user's manual. San Diego, Calif: Author, 1980.

Thonndyke, P. W., \& Hayes-Roth, B. Differences in spatial knowledge acquired from maps and navigation. Cognitive Psychology, 1982, 14, 560-589.

Thonndyke, P. W., \& Stasz, C. Individual differences in procedures for knowledge acquisition from maps. Cognitive Psychology, 1980, 12, 137-175. 